\title{
Laser-induced bipolar electrochemistry---on-demand formation of bipolar electrodes in a solid polymer light-emitting electrochemical cell
}

\author{
Dr. Faleh AlTal and Prof. Jun Gao* \\ Department of Physics, Engineering Physics and Astronomy, Queen's University, Kingston, Ontario, K7L 3N6, Canada \\ KEYWORDS: Bipolar electrochemistry, light emitting electrochemical cells, conjugated polymers, electrochemical doping, \\ photoluminescence quenching.
}

\begin{abstract}
Bipolar electrochemistry (BPEC) is a versatile and powerful technique that has found applications in sensing, chemical synthesis, catalysis, fuel cells and batteries, among others. In BPEC, the reactions of interest occur at a wireless, bipolar electrode (BPE). BPEC is most commonly carried out in an electrochemical cell that contains an electrolyte solution, in which a metallic BPE is immersed and polarized when the wired driving electrodes are biased. In this article, we demonstrate BPEC in a solid light-emitting electro-chemical cell (LEC) that does not initially contain a BPE. Shining a focused laser beam onto the mixed conductor LEC film causes the illuminated spot to function as a BPE from which redox reactions are induced and visualized. Separate experiments using a photosensitizer (widely used in polymer solar cells) confirm that a BPE is formed on-demand via photo-absorption that causes the illuminated spot to have elevated photo-conductivity. The simplicity of laser-induced BPEC offers exciting opportunities to explore sciences and applications of BPEC in the new realm of solid-state organic photonic devices.
\end{abstract}

In an electrochemical cell, a bipolar electrode (BPE) is a wireless conductor separated from a wired driving electrode (DE) by an electrolyte solution. The "bipolar" name stems from the BPE's ability to simultaneously induce reduction and oxidation reactions at its two opposite poles when the electrochemical cell is polarized.1-2 BPEs are particularly useful in applications and mechanistic studies that are simply impossible to realize with a conventional wired electrode. For example, "micro-swimmers" are created when suspended micro-BPEs are propelled by bubbles generated asymmetrically at the poles of the BPEs. ${ }^{3-5}$ Electrochemiluminescence (ECL) can be generated in the entire volume of an electrolyte solution impregnated with millions of micro-BPEs, each remotely addressed by bipolar electrochemistry (BPEC) and acts as an individual micro-emitter. ${ }^{6}$ BPEC is also efficient at synthesizing Janus particles with various combinations of micro-BPE and deposition materials in bulk, ${ }^{7}$ or producing materials with a compositional gradient. 8-12 The versatility of BPEC is further evidenced by its application, mainly in the form of BPE arrays, in high throughput screening and detection of catalysts or biomolecules. ${ }^{13-20}$

The aforementioned BPEC applications mainly utilize metal or carbon (fiber, beads, nanotubes or graphene) BPEs immersed in a liquid electrolyte. Recently, BPEC research has acquired a new, solid-state platform in lightemitting electrochemical cells (LECs). ${ }^{21}$ LECs are organic EL devices first developed in the 90s to overcome the severe limitations of organic light-emitting diodes (OLEDs). ${ }^{22}$ LECs often employ the same light emitters as those used in
OLEDs, which can be conjugated polymers (CPs), small organic molecules or transition metal complexes. ${ }^{23-26} \mathrm{Un}$ like OLEDs, however, the active layer of an LEC is a mixed ionic/electronic conductor. The archetypical PLEC, for example, contains both a solid polymer electrolyte (SPE) as well as a light-emitting CP. ${ }^{22}$ LECs are fundamentally different from OLEDs in that the mixed conductor active layer is electrochemically $\mathrm{p}$ - and $\mathrm{n}$-doped in situ during operation. Light emission in an LEC occurs when the expanding $\mathrm{p}$ - and $\mathrm{n}$-doped regions meet to form a $\mathrm{p}$-n homojunction. ${ }^{27}$

With two easily accessible built-in indicators in photoluminescence (PL) and electroluminescence (EL), LECs are supremely suited for mechanistic BPEC research. New BPE types, such as serially connected light-emitting p-n junctions, and electrochemically doped CPs, have been demonstrated in polymer LECs (PLECs). ${ }^{28-29}$ BPEC, in return, allows for new designs and functionalities to realize high performance LECs and photovoltaic devices. For example, BPE arrays, in both open and closed configurations, significantly alter the turn-on response and emission profiles of PLECs. ${ }^{28,30}$ A bulk homojunction is created when thousands of conductive indium tin oxide or metallic micro-particles are dispersed into the PLEC active layer. ${ }^{30-32}$ Bulk homojunction PLECs exhibit 3D EL that is orders of magnitude more intense than a single-junction PLEC. Moreover, bulk homojunction PLECs exhibit a giant open-circuit voltage when operated as a photovoltaic cell.

Doping affects both the electrical and optical properties of the LEC active layer. First, it renders the active layer highly conductive and the DE/CP contacts ohmic, making it 
possible to fabricate planar (vs. sandwich) LECs with a large separation between the DEs. ${ }^{33-34}$ A schematic of a planar PLEC is shown in Figure 1(a). Second, doping strongly quenches the $\mathrm{PL}$ of the $\mathrm{CP}$, making it possible to monitor the dynamic doping process in real-time using optical probes. ${ }^{35-37}$ In this study, we fully exploit these two effects to demonstrate the world's first laser-induced BPE in the world's largest planar PLECs. We show that by shining a focused laser beam on a mixed polymeric conductor, the increase in conductivity as a result of photo-absorption is enough to induce redox doping reactions in a biased PLEC without any pre-fabricated BPE. The observation is corroborated with a phenomenological model based on optical-induced permittivity changes in the polymer semiconductor. The discovery is further extended to a material system commonly used in polymer bulk heterojunction solar cells. We show that BPEs can be activated ondemand, with a beam of light anywhere in the exposed PLEC.

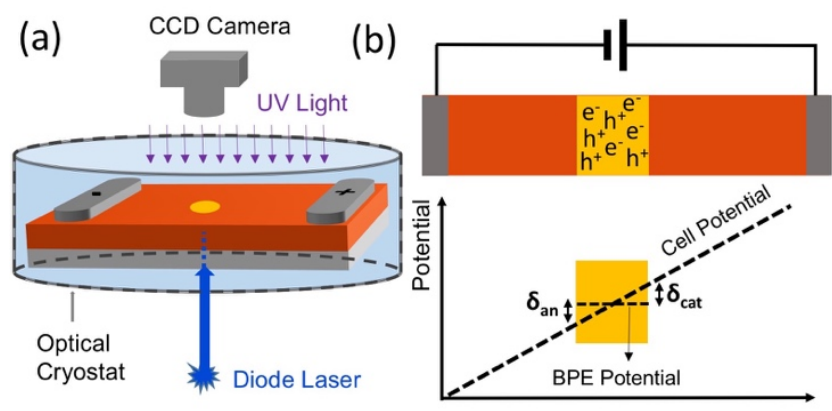

Figure 1. (a) Schematic of the experimental set-up and a planar PLEC fabricated on a glass substrate. The pair of the driving electrodes, coated on top of the polymer film, are separated by $11 \mathrm{~mm}$. The planar PLEC was tested in an optical cryostat under vacuum. The planar PLEC was illuminated from the bottom using a blue diode laser and imaged from above under UV illumination. (b) Schematic of a biased planar PLEC including a BPE. The lower part shows the electric potential distribution across the PLEC. Note that the BPE in this illustration is idealized as an equi-potential body.

\section{EXPERIMENTAL DETAILS}

The active layer of the PLEC is comprised of three materials: a $\mathrm{CP}$, polyethylene oxide (PEO, $\mathrm{Mw}=400 \mathrm{~K})$ and potassium trifluoromethanesulfonate (KTf). The solid complex of PEO and KTf constitutes a polymer electrolyte containing solvated $\mathrm{K}^{+}$and $\mathrm{CF}_{3} \mathrm{SO}_{3}{ }^{-}$ions. Both PEO and $\mathrm{KTf}$ were purchased from Aldrich and used as received. The CP is poly[2-methoxy-5-(2-ethylhexyloxy)-1,4-

phenylenevinylene], or MEH-PPV, an orange emitter sourced from OLEDKing Optoelectronic Materials Ltd, China with a molecular weight of $\mathrm{Mw}=3.3 \times 10^{5}$ and a polydispersity index of 1.4. MEH-PPV, PEO and KTf were dissolved in cyclohexanone with concentrations of $1 \mathrm{wt} \%$ (10 $\mathrm{mg}$ of solid per $1 \mathrm{ml}$ of solvent), $5 \mathrm{wt} \%$ and $0.24 \mathrm{wt} \%$, respectively. Suitable amounts of the three solutions were mixed to prepare a solution with a weight ratio of $\mathrm{MEH}$ PPV:PEO:KTf = 100:125:12. $100 \mu \mathrm{l}$ of this final solution was spin cast onto a glass substrate at $2500 \mathrm{rpm}$ for $60 \mathrm{~s}$ followed by $120 \mathrm{~s}$ at $4000 \mathrm{rpm}$. The cast film was left on a hot plate to dry at $50{ }^{\circ} \mathrm{C}$ for five hours. Afterwards, the cast film was annealed at $100{ }^{\circ} \mathrm{C}$ for 30 minutes, followed by rapid cooling on a large copper block. The polymer film thickness was measured with a DekTak stylus profiler. Finally, the PLEC was completed with the deposition of aluminum driving electrodes on top of the polymer film. A pair of Al driving electrodes $100 \mathrm{~nm}$ in thickness was thermally evaporated through a shadow mask under high vacuum $\left(10^{-6} \mathrm{mbar}\right)$. All solution making, spin casting of polymer films and thermal deposition of metal electrodes was carried out in a MBraun integrated glove box/evaporator system filled with dry nitrogen.

The planar PLECs were tested in a low-profile microscopy cryostat under vacuum. The PLECs were heated to 360 $\mathrm{K}$ in order to promote ion motion during operation. The vacuum chamber provides protection from the environment and optical access to both the top and bottom sides of the PLEC, as shown in Figure 1(a). A blue laser diode emitting at $473 \mathrm{~nm}$ was used as an excitation source. The laser beam was coupled into a single mode optical fiber using a coupling lens. The output of the fiber was collimated using a Thorlabs fixed focus collimation lens package (F280SMAA) and steered towards the bottom of the substrate using a mirror. The beam was then focused on the active film using a six-centimeter focal length lens that was attached to a zaxis translation stage to control the laser spot size on the film. A computer-controlled CCD camera, mounted on the viewing port of a Nikon fluorescence microscope, was used to image the device. The device was uniformly illuminated from the top using a UV ring light. A Keithley 237 source measurement unit was used to drive the PLEC with a bias voltage of $300 \mathrm{~V}$ or $500 \mathrm{~V}$. The device temperature was maintained to within $0.1 \mathrm{~K}$ via a Cryocon $32 \mathrm{~B}$ temperature controller.

\section{RESULTS}

The planar PLECs of this study had an $11 \mathrm{~mm}$ separation between the DEs and a width of $15 \mathrm{~mm}$. These are the largest planar PLECs ever reported in terms of the interelectrode gap size. The basic premise of a laser-induced BPE in a planar PLEC is shown in Figure 1(b). When the voltage bias was first applied to the DEs, the electric potential could be modelled approximately by a linear function of distance between the DEs. In an interior region illuminated with a beam of laser, however, photo-generated electrons and holes in MEH-PPV elevate the local conductivity of the polymer film. If the photoconductivity of the illuminated region is sufficiently high, the potential drop in the region should decrease and approach that of an equipotential conductor. Thus, we expect to observe doping reactions at the extremities of the illuminated regions, where the potential difference between the illuminated region and the non-illuminated region is the largest. In the following discussion, we call such a laser-induced BPE a laser-BPE for short. Realistically, we expect any doping reactions induced by a laser-BPE to be weaker in comparison to those induced by a pre-fabricated metallic BPE. This is because the photoconductivity of the PLEC film, which also contained over $50 \%$ of PEO, cannot match the conductivity of a metal. Further complicating matters are the fast propagating DE $\mathrm{p}$ - and n-doping, which can easily overwhelm and obscure any doping generated by the laser-BPE upon reaching the illuminated region. For this reason, the DE separation was designed to be the largest possible (11 $\mathrm{mm}$ ) for the given substrate size to delay this encounter. 
Many other factors, such as bias voltage, operating temperature, imaging conditions, laser beam size and intensity, PLEC film composition and illumination direction (top vs. bottom) were also experimented. After many trials, a successful observation was realized and is shown below.

Figure 2(a) shows a slice (1.3 $\mathrm{mm}$ in height) of the 11 $\mathrm{mm}$ planar PLEC containing the illuminated spot in two consecutive images taken less than $2 \mathrm{~s}$ apart. One image has the laser turned on and one has the laser turned off. The images were taken when the PLEC had been under bias for about $133 \mathrm{~s}$. The positive and negative DE edges are indicated by the vertical dashed lines. The polymer film exhibits the red/orange PL from MEH-PPV under UV illumination from the top. The illuminated spot is clearly visible against the background PL. In these images, significant PL quenching is visible next to the DEs. The p-doped region next to the positive DE is much darker than the n-doped region next to the negative $\mathrm{DE}$. We note that the $\mathrm{DE} \mathrm{p}$ - and n-doping fronts are still a large distance away from the illuminated spot. Faint, finger-like darkened region is also visible to the left of the illuminated spot, extending in the direction of the applied electric field. This is the p-doping reaction expected from the laser-BPE. An enhanced view of the region containing the illuminated spot is shown in Figure 2(b). The intensity profile of the excitation laser, measured with the CCD camera, is overlaid on the lower image. $100 \mu \mathrm{W}$ of laser power was delivered to a Gaussian spot with a $1 / e^{2}$ diameter of $200 \mu \mathrm{m}$. This gives a peak laser intensity of $6.25 \mathrm{~mW} / \mathrm{mm}^{2}$. Comparing the two images in Figure 2(b), we observe that the dark finger initially originated at a distance away from the center of the laser spot, as it should be for a BPE-induced doping reaction. In other words, the laser-BPE must have a non-zero diameter. The displacement between the laser beam center and the right edge of the BPE doping was determined to be $90 \pm 20$ $\mu \mathrm{m}$. The uncertainty of $20 \mu \mathrm{m}$ is attributed to the pixel size in the device images. This gives a Laser-BPE diameter of $180 \mu \mathrm{m}$. The presence of BPE p-doping is confirmed in Figure 2(c), which shows eight time-lapsed images. The dark finger increases in length with time, behaving exactly as that from a metallic disc BPE. N-doping, however, was not observed from the opposite end of the laser-BPE. The possible cause for this absence will be discussed later.

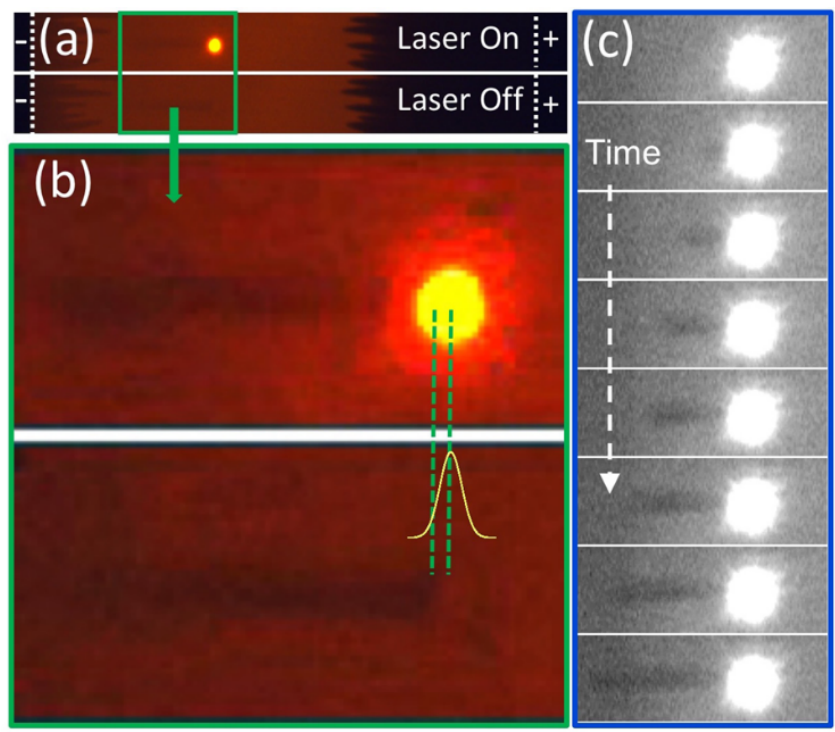

Figure 2. (a) Original photoluminescence images of the planar PLEC under test about $133 \mathrm{~s}$ after a $500 \mathrm{~V}$ bias had been applied, with and without laser illumination. (b) Expanded view of the region enclosed by the green box in (a). The enclosed region had been enhanced in Photoshop by applying levels adjustment filter (0-0.69-100). The measured laser beam Gaussian profile was overlaid on the image in the bottom image. The vertical dashed lines denote the locations of the laser beam center and the right edge of the BPE doping. (c) Time-lapsed images of the planar PLEC in greyscale. The same levels adjustment Photoshop filter was applied to all images (49-1.61-104). The time delay between consecutive images was $13.7 \mathrm{~s}$.

To elucidate the phenomenon shown in Figure 2, we performed COMSOL simulation to determine the electric potential distribution across the laser-BPE. The photoconductivity of a CP such as MEH-PPV is proportional to the photo-excitation level. Conductivity (at frequency $\omega$ ) manifests itself as an imaginary part in the complex relative permittivity constant of the material according to:

$\varepsilon=\varepsilon^{\prime}+j \varepsilon^{\prime \prime}=\varepsilon^{\prime}+j \frac{\sigma}{\omega \varepsilon_{0}}$

Equation (1)

Where $\varepsilon^{\prime}$ and $\varepsilon^{\prime \prime}$ are the real and imaginary parts of the relative permittivity, $\sigma$ is the frequency dependent conductivity and $\varepsilon_{0}$ is the vacuum permittivity. In our case, the conductivity arises from photo-excitation and generation of free carriers. The photoconductivity of MEH-PPV has been shown experimentally to be linearly proportional to light intensity. ${ }^{38}$ Hence, we can rewrite the imaginary part of the relative permittivity in terms of the excitation beam intensity, Gaussian in our case, as the following:

$\varepsilon^{\prime \prime}=\varepsilon_{p}^{\prime \prime} e^{-8\left|\rho-\rho_{0}\right|^{2} / W_{B}^{2}}$

Equation

where $\varepsilon_{p}^{\prime \prime}$ is the imaginary part of the relative permittivity at the point of peak laser intensity, $\rho$ is the radial coordinate in a cylindrical system, $\rho_{0}$ is the radial coordinate of the beam center and $W_{B}$ is the $1 / e^{2}$ Gaussian beam width. Note that in Equation (1) $\varepsilon^{\prime \prime}$ becomes infinite as $\omega$ approaches zero. Here, we assume that $\varepsilon^{\prime \prime}$ is finite at an arbitrarily low frequency. The spatial profile of $\varepsilon^{\prime \prime}$ as quantified by Equation (2) is then implemented in COMSOL to calculate the potential profile in the PLEC under bias. (a)

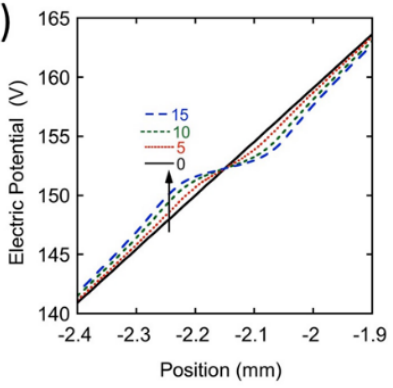

(b)

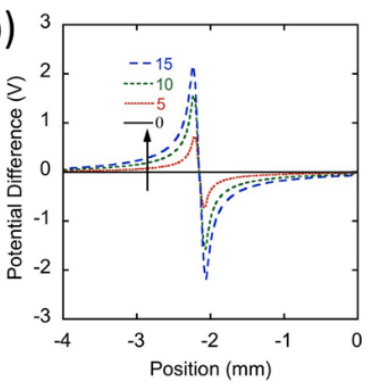

Figure 3. (a) The electric potential profile of the biased planar PLEC in the vicinity of the illuminated spot for different values of peak relative permittivity imaginary part $\left(\varepsilon_{p}^{\prime \prime}\right)$ shown in the legend. These potential profiles were calculated in COMSOL by solving Poisson's equation in a charge-free domain and boundary conditions set by the DEs voltage bias. (b) The potential difference profile calculated by subtracting the 
potential that corresponds to $\varepsilon_{p}^{\prime \prime}=0$ from the potential profile with $\varepsilon_{p}^{\prime \prime}$ assigned the values in the legend.

Figure 3(a) shows the 1D potential distribution across the PLEC in the direction of the applied electric field passing through the laser-BPE center for different values of $\varepsilon_{p}^{\prime \prime}$. When $\varepsilon_{p}^{\prime \prime}$ is zero, the PLEC is a pure dielectric. The electric potential increases linearly from the negative DE toward the positive DE. When the laser is turned on, photogeneration of charged carriers contributes to a non-zero $\varepsilon^{\prime \prime}$ that is described by Equation (2). We observe that the potential line is flattened at the illuminated spot as $\varepsilon_{p}^{\prime \prime}$ increases. This is an expected behavior-when the CP becomes more conductive it will behave more like an equipotential conductor. In Figure 3(b), the same data is replotted with the zero $\varepsilon_{p}^{\prime \prime}$ potential line subtracted from the potential profiles under laser illumination. This rendering of the data produced pairs of positive and negative peaks in potential difference whose height and separation increase with $\varepsilon_{p}^{\prime \prime}$.

The recovery of these positive (anodic) and negative (cathodic) peaks in potential difference justifies the observation of redox reactions at the extremities of a laser-BPE. A value of $\varepsilon_{p}^{\prime \prime}=15$ produces a $90 \mu \mathrm{m}$ separation between the anodic or cathodic peak and the BPE center that matches the experimentally observed separation of $90 \mu \mathrm{m}$ shown in Figure 2(b). The validity of this analysis is further enhanced by the retrieval of a peak-to-peak potential drop of $4.35 \mathrm{~V}$ at this $\varepsilon_{p}^{\prime \prime}$ value, which satisfies the minimum requirement to induce redox reaction in MEH-PPV which has an energy gap of $2.4 \mathrm{eV} .{ }^{39}$

In the creation of a laser-BPE, the CP is responsible for reaching the necessary conductivity of a BPE upon photoexcitation. Although MEH-PPV is a photoconductive material, the photo-generated charge carriers recombine quickly via both radiative and non-radiative pathways. This is evidenced by its poor photoelectron quantum yield $(\sim 10$ 3). ${ }^{40}$ Therefore, it is very difficult and quite remarkable to have observed any doping reaction at the illuminated region. The photoconductivity of MEH-PPV can be dramatically enhanced by adding a stronger electron acceptor such as the buckminsterfullerene, C60. ${ }^{41}$ In the MEH-PPV:C60 composite, photo-generated electrons in MEH-PPV are captured, in less than a picosecond, by the low-lying LUMO of PCBM, causing effective ( 100\%) separation of the photo-generated electrons and holes and suppression of early recombination. In fact, it is the discovery of this ultrafast photo-induced charge transfer between conjugated polymers and fullerenes that gave birth to the highly efficient polymer bulk-heterojunction solar cells. ${ }^{42-43}$ We apply this concept to demonstrate that photoconductivity is indeed at play in the creation of a Laser-BPE.

A chlorobenzene solution containing equal weight amounts of MEH-PPV and PCBM was prepared. PCBM, $[6,6]$-phenyl- $\mathrm{C}_{61}$-butyric acid methyl ester, is a soluble $\mathrm{C} 60$ derivative commonly used in polymer solar cells. Small droplets of the MEH-PPV:PCBM solution were drop-cast to the exposed surface of a planar PLEC identical to the one shown in Figure 2. The droplets were allowed to dry on a hot plate at $50^{\circ} \mathrm{C}$ for two hours before the PLEC was activated. Figure 4(a) shows time-lapse images of the planar
PLEC under a $300 \mathrm{~V}$ bias and UV illumination from the top. The images only show a region that contained two $\mathrm{MEH}$ PPV:PCBM dots, one illuminated with the laser and one without illumination. The illuminated MEH-PPV dot had a diameter of $1.04 \mathrm{~mm}$. The $100 \mu \mathrm{W}$ laser beam was expanded to $730 \mu \mathrm{m}$ in diameter to illuminate the dot from below. This means that the average beam intensity was about 13 times less than that in the previous device without any MEH-PPV:PCBM dots. On the other hand, it has been shown that at a one-to-one MEH-PPV: $\mathrm{C}_{60}$ ratio, the steadystate photoconductivity enhancement at $473 \mathrm{~nm}$ was about 20 times higher compared to pure MEH-PPV. ${ }^{41}$ Therefore, the free carrier concentration is comparable to the illuminated region in Figure 2.

In Figure 4(a), the top MEH-PPV:PCBM dot was not illuminated by laser. It appeared black under UV illumination due to heavy PL quenching. Note that the PL quenching by PCBM or $\mathrm{C60}$ is different from that caused by in situ electrochemical doping. In the former case, the PL is quenched due to the afore-mentioned photo-induced charge transfer process, also known as photo-doping. The lower dot is laser-illuminated form the bottom of the cell. Remarkably, a dark, growing PL-quenched region in the shape of a finger appeared on the left (anodic) side of the illuminated dot. There is little doubt that the dark finger is caused by electrochemical p-doping originated from the laser-BPE. Compared to the laser-BPE doping shown in Figure 2, the finger here is much darker and highly visible without any enhancement. However, unlike the previous case, the actual size of the laser-BPE cannot be observed by turning off the laser due to the presence of PCBM that causes heavy PL quenching throughout the MEH-PPV:PCBM dot.
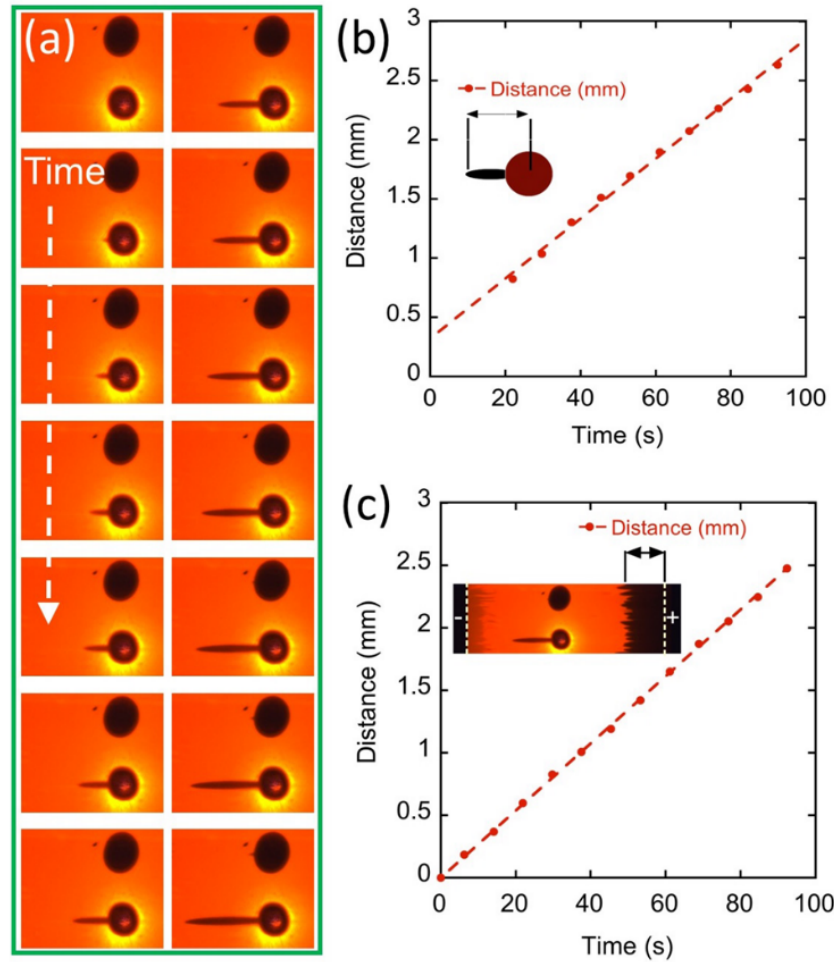

Figure 4. (a) Original time-lapsed photoluminescence images of a planar PLEC in the region that contained two MEHPPV:PCBM (1:1) dots coated on top of the PLEC film. The bottom dot was illuminated with the blue diode laser from below. 
The time delay between consecutive images was $7.8 \mathrm{~s}$. (b) Distance between tip of the propagating laser-BPE p-doping to the center of the illuminated MEH-PPV:PCBM dot as a function of time. The data were extracted from 10 images in (a). The dashed line is a linear fit to the data. The schematic shows how the distance was measured. (c) Average distance between the propagating DE p-doping and the edge of the positive DE. The dashed line is a linear fit to the data. The schematic shows electrode-to-electrode view of the PLEC and how the distance was measured. The distance value (averages) were extracted in Matlab.

To determine the size of the Laser-BPE, the doping fingertip positions (relative to the dot center) were extracted from the images and plotted against time, as shown in Figure 4(b). The relation is highly linear, from which a propagation rate of $25.3 \mu \mathrm{m} / \mathrm{s}$ was calculated. Time zero is taken as the time when p-doping was first observed from the positive DE electrode. The $y$ (position axis) intercept of about $320 \mu \mathrm{m}$ gives the radius of the laser-BPE. For comparison, Figure 4(c) shows the average DE p-doping front position (relative to the edge of the positive DE) as a function of time. A linear relation is again observed, from which a slightly fast propagation rate of $26.8 \mu \mathrm{m} / \mathrm{s}$ was deduced. A tiny protrusion is also visible from the top $\mathrm{MEH}$ PPV:PCBM dot after a significant delay. The protrusion increased in size and pointed to the direction of the negative DE. This suggests that it is also electrochemical pdoping in nature. Thus, the top dot also functioned as a BPE, albeit a much weaker one without laser illumination.

The experimental and modeling results of Figure 2 and Figure 3 offer a proof-of-concept demonstration of a new type of BPE. BPE illumination has been reported in some recent studies where light is applied as a stimulus to facilitate various photoelectrochemical actions. ${ }^{44-47}$. In particular, direct UV illumination of $\mathrm{TiO}_{2}$ nanofibers or nanoparticles allows for the generation of Janus objects via electric field assisted photoelectrochemistry. ${ }^{48-49}$ The current study represents a major advancement through its demonstration of BPE from illumination alone. The laser spot defines the size and location of the BPE generated. The laser-BPE can be created on-demand, anywhere between the DEs, and without the use of any metallic or sensitizing materials. This is made possible with the mixed conductor of a PLEC. In a planar PLEC, the mixed conductor provides both the counter-ions needed for the doping reaction and a $\mathrm{CP}$ that serves the dual functions of a photoconductor and the species to be reduced/oxidized. In Figure 4, the concept of laser-BPE is extended to a well-known polymer solar cell material with greatly enhanced photosensitivity. This resulted in faster and stronger doping reaction from the illuminated Laser-BPE at a lower excitation level.

In both Figure 2 and Figure 4, the expected electrochemical $n$-doping from the cathodic pole of the Laser-BPE is notably absent. Whereas in planar PLECs with evaporated or inkjet-printed metal disc BPEs, both $\mathrm{p}$ - and n-doping have been observed under the right conditions. In planar PLECs made with metallic BPE arrays, the $\mathrm{p}$ - and n-doping originated from neighboring BPEs can even interact to form strongly emitting light-emitting p-n junctions. ${ }^{28} \mathrm{We}$ note that even for metallic BPEs, the appearance of BPE doping can be significantly delayed due to an insufficient applied driving potential. ${ }^{21}$ Moreover, the delays incurred by the BPE p- and n-doping can also be different. In one instance, only BPE p-doping was observed even when the DE doping fronts had already reached the BPE. In order to satisfy the fundamental requirement of electric neutrality for any BPE, we must consider the following possibilities: (1) the BPE n-doping is simply too faint and small in size to be imaged under the testing conditions. In planar PLECs, the electrochemical n-doping of a CP is typically slower and fainter (less quenched) in appearance than p-doping, making it difficult to observe. This can be seen in Figure 2(a) by comparing the DE n-doping on the left with the dark DE p-doping to the right. Near the laser-BPE, the faint n-doping can easily be obscured by the strong laserinduced PL near the edge of the BPE or the dark MEHPPV:PCBM composite. (2) the BPE itself or the electrolyte has participated in the redox reaction. This has been confirmed in the doping reactions from BPEs that are electrochemically $\mathrm{p}$ - or $\mathrm{n}$-doped polymers. ${ }^{29}$ In a BPE that is an electrochemically $\mathrm{p}$-doped MEH-PPV, only $\mathrm{p}$-doping was observed while the cathodic reaction took the form of dedoping of a p-doped BPE. In the MEH-PPV:PCBM composite Laser-BPE, the non-illuminated PCBM near the top surface could be easily reduced due to its low-lying LUMO. For the well illuminated regions near the bottom of the film, photo-doping should dominate.

To test the first possibility, the laser power was increased to $400 \mu \mathrm{W}$ while the beam diameter decreased to $367 \mu \mathrm{m}$ to test a planar PLEC with a MEH-PPV:PCBM disc of $1.15 \mathrm{~mm}$ in diameter. The time-lapse images of this device are shown in Figure S1 of the Supporting Information section. In the post-processed images, a small bright protrusion is visible directly to the right of the illuminated MEH-PPV:PCBM disc. This is consistent with the appearance of BPE n-doping at the cathodic pole of the laser-BPE. The faint $n$-doping is only visible when $\mathrm{p}$-doping from the positive DE had already reached the laser-BPE. This prevented the $n$-doped region from propagating but made it more visible due to the dark appearance of the surrounding p-doped region. Eventually, the n-doping dot faded out. This ruled out the possibility that the dot was a defect in the film.

\section{CONCLUSION}

We demonstrate a new type of BPEs in a solid-state electrochemical cell by localized laser illumination only. This was realized in a planar PLEC that did not initially contain a physical BPE. Shining a focused laser beam onto the mixed conductor PLEC film causes the illuminated area to acquire sufficient photoconductivity to function as a laserBPE. The planar PLEC, with a fully exposed surface between the DEs, also allows for easy visualization of the dynamic doping reaction induced by the laser-BPE. Modeling and additional experiments with a more efficient photoconductor prove beyond doubt the formation of a laserBPE via photo-excitation. The simplicity of laser-induced BPEC promises exciting applications in solid-state organic photonic devices. We envision the formation of multiple laser-BPEs of various sizes and shapes, via the manipulation of the excitation beam or beams and the use of a highly photoactive under-layer below the solid-state electrochemical cell. The concept of laser-BPE can also be used as a visual platform to screen photoconductors for photovol- 
taic applications. BPEC is poised to have a greater impact due to its intrinsic advantages and new opportunities offered by a solid-state electrochemical cell.

\section{SUPPORTING INFORMATION}

FigureS1 shows evidence of n-doping induced by a laser-BPE operated under high laser illumination intensity.

\section{AUTHOR INFORMATION}

\section{Corresponding Author}

*jungao@queensu.ca

\section{Author Contributions}

The manuscript was written through contributions of all authors.

\section{ACKNOWLEDGMENT}

This research was supported by the Natural Sciences and Engineering Research Council of Canada (Grant Number 2502442010). Faleh AlTal is grateful for the support of an Ontario Trillium Scholarship.

\section{ABBREVIATIONS}

BPEC, Bipolar electrochemistry; BPE, bipolar electrode; LEC, light-emitting electrochemical cell; DE, driving electrode; ECL, Electrochemiluminescence; OLED, organic light-emitting diode; CP, conjugated polymer; SPE, solid polymer electrolyte; PL, photoluminescence; EL, electroluminescence; PLEC, polymer light-emitting electrochemical cell; PEO, polyethylene oxide; KTf, potassium trifluoromethanesulfonate; MEH-PPV, poly[2-methoxy-5-(2-ethylhexyloxy)-1,4-phenylenevinylene]; PCBM, [6,6]-phenyl-C61-butyric acid methyl ester

\section{REFERENCE}

1. Loget, G.; Zigah, D.; Bouffier, L.; Sojic, N.; Kuhn, A., Bipolar Electrochemistry: From Materials Science to Motion and Beyond. Accounts of Chemical Research 2013, 46 (11), 2513-2523.

2. Fosdick, S. E.; Knust, K. N.; Scida, K.; Crooks, R. M., Bipolar Electrochemistry. Angewandte Chemie-International Edition 2013, 52 (40), 10438-10456.

3. Fattah, Z.; Loget, G.; Lapeyre, V.; Garrigue, P.; Warakulwit, C.; Limtrakul, J.; Bouffier, L.; Kuhn, A., Straightforward single-step generation of microswimmers by bipolar electrochemistry. Electrochimica Acta 2011, 56 (28), 10562-10566.

4. $\quad$ Jiang, J. Z.; Guo, M. H.; Yao, F. Z.; Li, J.; Sun, J. J., Propulsion of copper microswimmers in folded fluid channels by bipolar electrochemistry. Rsc Advances 2017, 7 (11), 6297-6302.

5. Sentic, M.; Loget, G.; Manojlovic, D.; Kuhn, A.; Sojic, N., LightEmitting Electrochemical "Swimmers". Angewandte Chemie-International Edition 2012, 51 (45), 11284-11288.

6. Sentic, M.; Arbault, S.; Bouffier, L.; Manojlovic, D.; Kuhn, A.; Sojic, N., 3D electrogenerated chemiluminescence: from surface-confined reactions to bulk emission. Chem. Sci. 2015, 6 (8), 4433-4437.

$7 . \quad$ Loget, G.; Roche, J.; Kuhn, A., True Bulk Synthesis of Janus Objects by Bipolar Electrochemistry. Advanced Materials 2012, 24 (37), 5111-5116.

8. Ishiguro, Y.; Inagi, S.; Fuchigami, T., Gradient Doping of Conducting Polymer Films by Means of Bipolar Electrochemistry. Langmuir 2011, 27 (11), 7158-7162.

9. $\quad$ Lundgren, A.; Munktell, S.; Lacey, M.; Berglin, M.; Bjorefors, F., Formation of Gold Nanoparticle Size and Density Gradients via Bipolar Electrochemistry. ChemElectroChem 2016, 3 (3), 378-382.

10. Ramakrishnan, S.; Shannon, C., Display of Solid-State Materials Using Bipolar Electrochemistry. Langmuir 2010, 26 (7), 4602-4606.
11. Tan, S. M.; Pumera, M., Composition-Graded MoWSx Hybrids with Tailored Catalytic Activity by Bipolar Electrochemistry. Acs Applied Materials \& Interfaces 2017, 9 (48), 41955-41964.

12. Inagi, S., Fabrication of gradient polymer surfaces using bipolar electrochemistry. Polymer Journal 2016, 48 (1), 39-44.

13. Xiao, Y.; Xu, L. R.; Qi, L. W., Electrochemiluminescence bipolar electrode array for the multiplexed detection of glucose, lactate and choline based on a versatile enzymatic approach. Talanta 2017, 165, 577-583.

14. Khoshfetrat, S. M.; Ranjbari, M.; Shayan, M.; Mehrgardi, M. A.; Kiani, A., Wireless Electrochemiluminescence Bipolar Electrode Array for Visualized Genotyping of Single Nucleotide Polymorphism. Anal. Chem. 2015, 87 (16), 8123-8131.

15. Zhai, Q. F.; Zhang, X. W.; Han, Y. C.; Zhai, J. F.; Li, J.; Wang, E. K., A Nanoscale Multichannel Closed Bipolar Electrode Array for Electrochemiluminescence Sensing Platform. Anal. Chem. 2016, 88 (1), 945-951.

16. Termebaf, H.; Shayan, M.; Kiani, A., Two-Step Bipolar Electrochemistry: Generation of Composition Gradient and Visual Screening of Electrocatalytic Activity. Langmuir 2015, 31 (48), 13238-13246.

17. Lin, X.; Zheng, L.; Gao, G.; Chi, Y.; Chen, G., Electrochemiluminescence Imaging-Based High-Throughput Screening Platform for Electrocatalysts Used in Fuel Cells. Anal. Chem. 2012, 84 (18), 7700-7707.

18. Fosdick, S. E.; Berglund, S. P.; Mullins, C. B.; Crooks, R. M., Parallel Screening of Electrocatalyst Candidates Using Bipolar Electrochemistry. Anal. Chem. 2013, 85 (4), 2493-2499.

19. Munktell, S.; Nyholm, L.; Bjorefors, F., Towards high throughput corrosion screening using arrays of bipolar electrodes. Journal of Electroanalytical Chemistry 2015, 747, 77-82.

20. Yuan, F.; Qi, L.; Fereja, T. H.; Snizhko, D. V.; Liu, Z.; Zhang, W.; $\mathrm{Xu}, \mathrm{G}$., Regenerable bipolar electrochemiluminescence device using glassy

carbon bipolar electrode, stainless steel driving electrode and cold

patch. Electrochimica Acta 2018, 262, 182-186

21. Chen, S. L.; Wantz, G.; Bouffier, L.; Gao, J., Solid-State Bipolar Electrochemistry: Polymer-Based Light-Emitting Electrochemical Cells. ChemElectroChem 2016, 3 (3), 392-398.

22. Pei, Q. B.; Yu, G.; Zhang, C.; Yang, Y.; Heeger, A. J., Polymer Light-Emitting Electrochemical-Cells. Science 1995, 269 (5227), 1086 1088.

23. Gao, J., Polymer light-emitting electrochemical cells-Recent advances and future trends. Current opinion in Electrochemistry 2018, 7, 87-94.

24. Fresta, E.; Costa, R. D., Beyond traditional light-emitting electrochemical cells - a review of new device designs and emitters. Journal of Materials Chemistry C 2017, 5 (23), 5643-5675.

25. Tang, S.; Edman, L., Light-Emitting Electrochemical Cells: A Review on Recent Progress. Topics in Current Chemistry 2016, 374 (4).

26. Meier, S. B.; Tordera, D.; Pertegas, A.; Roldan-Carmona, C.; Orti, E.; Bolink, H. J., Light-emitting electrochemical cells: recent progress and future prospects. Materials Today 2014, 17 (5), 217-223.

27. Pei, Q.; Yang, Y.; Yu, G.; Zhang, C.; Heeger, A. J., Polymer LightEmitting Electrochemical Cells: In Situ Formation of a Light-Emitting p-n Junction. Journal of the American Chemical Society 1996, 118 (16), 3922. 3929.

28. Gao, J.; Chen, S. L.; AITal, F.; Hu, S. Y.; Bouffier, L.; Wantz, G., Bipolar Electrode Array Embedded in a Polymer Light-Emitting Electrochemical Cell. Acs Applied Materials \& Interfaces 2017, 9 (37), 32405-32410.

29. Hu, S. Y.; Chi, X.; Chen, S. L.; AlTal, F.; Gao, J., Visualizing the Bipolar Electrochemistry of Electrochemically Doped Luminescent Conjugated Polymers. Journal of Physical Chemistry C 2017, 121 (15), 8409-8415.

30. Tracy, C.; Gao, J., Polymer Bulk Homojunction Photonic Devices. Applied Physics Letters 2005, 87, 143502.

31. Tracy, C.; Gao, J., Polymer bulk homojunction light-emitting electrochemical cells. Journal of Applied Physics 2006, 100, 104503.

32. Bonnet, W.; Tracy, C.; Wantz, G.; Liu, G. J.; Gao, J., Bulk Electroluminescence from Conjugated Polymer Thin Films via the Formation of Gold Nanoislands. Small 2008, 4 (10), 1707-1710.

33. Gao, J.; Dane, J., Planar polymer light-emitting electrochemical cells with extremely large interelectrode spacing. Applied Physics Letters 2003, 83 (15), 3027-3029. 
35. Gao, J.; Dane, J., Visualization of electrochemical doping and light-emitting junction formation in conjugated polymer films. Applied Physics Letters 2004, 84 (15), 2778-2780.

36. AITal, F.; Gao, J., Scanning photocurrent and PL imaging of a frozen polymer p-i-n junction. Physica Status Solidi-Rapid Research Letters 2015, 9 (1), 77-81.

37. Jafari, M. J.; Liu, J.; Engquist, I.; Ederth, T., Time-Resolved Chemical Mapping in Light-Emitting Electrochemical Cells. Acs Applied Materials \& Interfaces 2017, 9 (3), 2747-2757.

$38 . \quad$ Lee, C. H.; Yu, G.; Moses, D.; Sariciftci, N. S.; WudI, F.; Heeger, A. J., Transient Photoconductivity of Meh-Ppv and Its Sensitization by C-60. Mol. Cryst. Liq. Cryst. Sci. Technol. Sect. A-Mol. Cryst. Liq. Cryst. 1994, 256, 745-750.

39. Holt, A. L.; Leger, J. M.; Carter, S. A., Electrochemical and optical characterization of $\mathrm{p}$ - and $\mathrm{n}$-doped poly[2-methoxy-5-(2-ethylhexyloxy)1,4-phenylenevinylene]. Journal of Chemical Physics 2005, 123 (4).

40. Moses, D.; Wang, J.; Yu, G.; Heeger, A. J., Temperatureindependent photoconductivity in thin films of semiconducting polymers: Photocarrier sweep-out prior to deep trapping. Physical Review Letters 1998, 80 (12), 2685-2688.

41. Lee, C. H.; Yu, G.; Moses, D.; Pakbaz, K.; Zhang, C.; Sariciftci, N. S.; Heeger, A. J.; WudI, F., Sensitization of the Photoconductivity of Conducting Polymers by C-60 - Photoinduced Electron-Transfer. Physical Review B 1993, 48 (20), 15425-15433.

42. Sariciftci, N. S.; Smilowitz, L.; Heeger, A. J.; Wudl, F., Photoinduced Electron-Transfer from a Conducting Polymer to Buckminsterfullerene. Science 1992, 258 (5087), 1474-1476.

43. Yu, G.; Gao, J.; Hummelen, J. C.; WudI, F.; Heeger, A. J., Polymer Photovoltaic Cells - Enhanced Efficiencies Via a Network of Internal DonorAcceptor Heterojunctions. Science 1995, 270 (5243), 1789-1791.

44. Essmann, V.; Zhao, F. Y.; Hartmann, V.; Nowaczyk, M. M. Schuhmann, W.; Conzuelo, F., In Operando Investigation of Electrical Coupling of Photosystem 1 and Photosystem 2 by Means of Bipolar Electrochemistry. Anal. Chem. 2017, 89 (13), 7160-7165.

45. Loget, G.; Li, G. Z.; Fabre, B., Logic gates operated by bipolar photoelectrochemical water splitting. Chemical Communications 2015, 51 (55), 11115-11118.

46. Dong, R. F.; Hu, Y.; Wu, Y. F.; Gao, W.; Ren, B. Y.; Wang, Q. L.; Cai, Y. P., Visible-Light-Driven BiOl-Based Janus Micromotor in Pure Water. Journal of the American Chemical Society 2017, 139 (5), 1722-1725.

47. Zhang, Q. L.; Dong, R. F.; Chang, X. Y.; Ren, B. Y.; Tong, Z., Spiropyran-Decorated Si02-Pt Janus Micromotor: Preparation and LightInduced Dynamic Self-Assembly and Disassembly. Acs Applied Materials \& Interfaces 2015, 7 (44), 24585-24591.

48. Ongaro, M.; Roche, J.; Kuhn, A.; Ugo, P., Asymmetric Modification of TiO2 Nanofibers with Gold by Electric-Field-Assisted Photochemistry. ChemElectroChem 2014, 1 (12), 2048-2051.

49. Tiewcharoen, S.; Warakulwit, C.; Lapeyre, V.; Garrigue, P.; Fourier, L.; Elissalde, C.; Buffiere, S.; Legros, P.; Gayot, M.; Limtrakul, J.; Kuhn, A., Anisotropic Metal Deposition on Ti02 Particles by Electric-FieldInduced Charge Separation. Angewandte Chemie-International Edition 2017, 56 (38), 11431-11435. 
Graphical Abstract

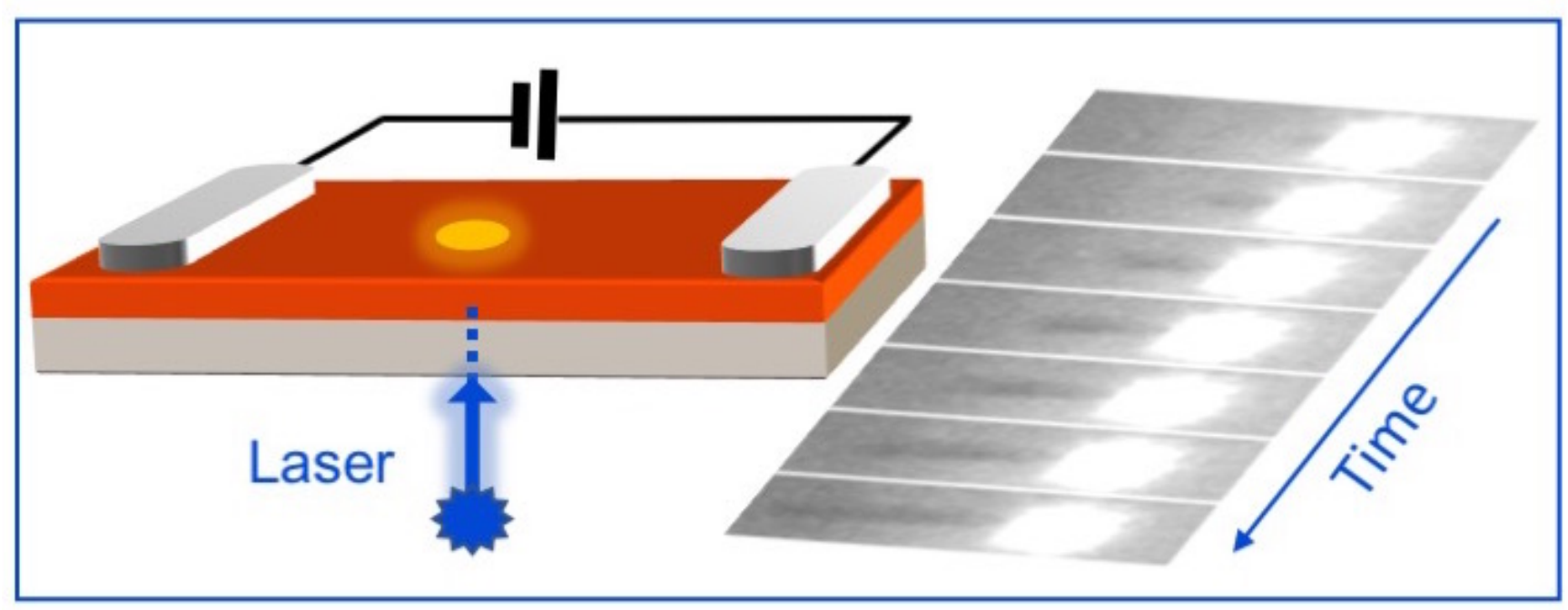

